\title{
Diabetic myonecrosis: challenges in diagnosis and management
}

\author{
KANYADA KOYSOMBAT, ${ }^{1 *}$ SARRA ELMUSTAFA, ${ }^{*}{ }^{*}$ HARDI MADANI, ${ }^{2}$ FELICITY KAPLAN ${ }^{3}$
}

Key words: diabetic myonecrosis; diabetic muscle infarction; diabetic nephropathy

\section{Introduction}

Diabetic myonecrosis (DMN) or diabetic muscle infarction is a rare complication of diabetes mellitus. To date, over half a century since first described in 1965, ${ }^{1}$ the pathogenesis and management approach is still incompletely understood. We describe a case of DMN, the multidisciplinary approach adopted and the challenges faced in the management of this patient.

\section{Case presentation}

A 29-year-old female presented with acute onset right thigh pain and swelling 24 hours after peritoneal dialysis catheter insertion. She had a background of type 1 diabetes mellitus diagnosed over 20 years prior to presentation. Her glycaemic control had been suboptimal, with multiple episodes of diabetic ketoacidosis and complications including diabetic retinopathy, gastroparesis, autonomic neuropathy and end stage renal disease secondary to diabetic nephropathy, requiring peritoneal dialysis. She was discharged after completing a course of intravenous antibiotics for presumed infection but re-presented one week later to her renal team with worsening right thigh pain and swelling. There was no history of trauma or symptoms to suggest an infective aetiology.

On examination she was in severe pain. She was tachycardic with a pulse rate of $110 \mathrm{bpm}$, blood pressure was elevated at $158 / 90 \mathrm{mmHg}$, she was afebrile and had normal oxygen saturation levels. The peritoneal catheter site appeared clean and her abdomen was soft with no ascites. The right thigh was markedly enlarged, circumference $45 \mathrm{~cm}$ compared with $25 \mathrm{~cm}$ on the left

\footnotetext{
Joint first authors

Specialist Registrar in Diabetes and Endocrinology, Department of Diabetes and Endocrinology, Lister Hospital, East and North Hertfordshire NHS Trust, Stevenage, UK

2 Consultant, Department of Radiology, Lister Hospital, East and North Hertfordshire NHS Trust, Stevenage, UK

3 Consultant in Diabetes and Endocrinology, Department of Diabetes and Endocrinology, Lister Hospital, East and North Hertfordshire NHS Trust, Stevenage, UK
}

Address for correspondence: Dr Kanyada Koysombat Department of Diabetes and Endocrinology, Lister Hospital, Coreys Mill Lane, Stevenage, Hertfordshire SG1 4AB, UK

E-mail: kanyada.koysombat@nhs.net

Br J Diabetes 2021;21:247-249

https://doi.org/10.15277/bjd.2021.311

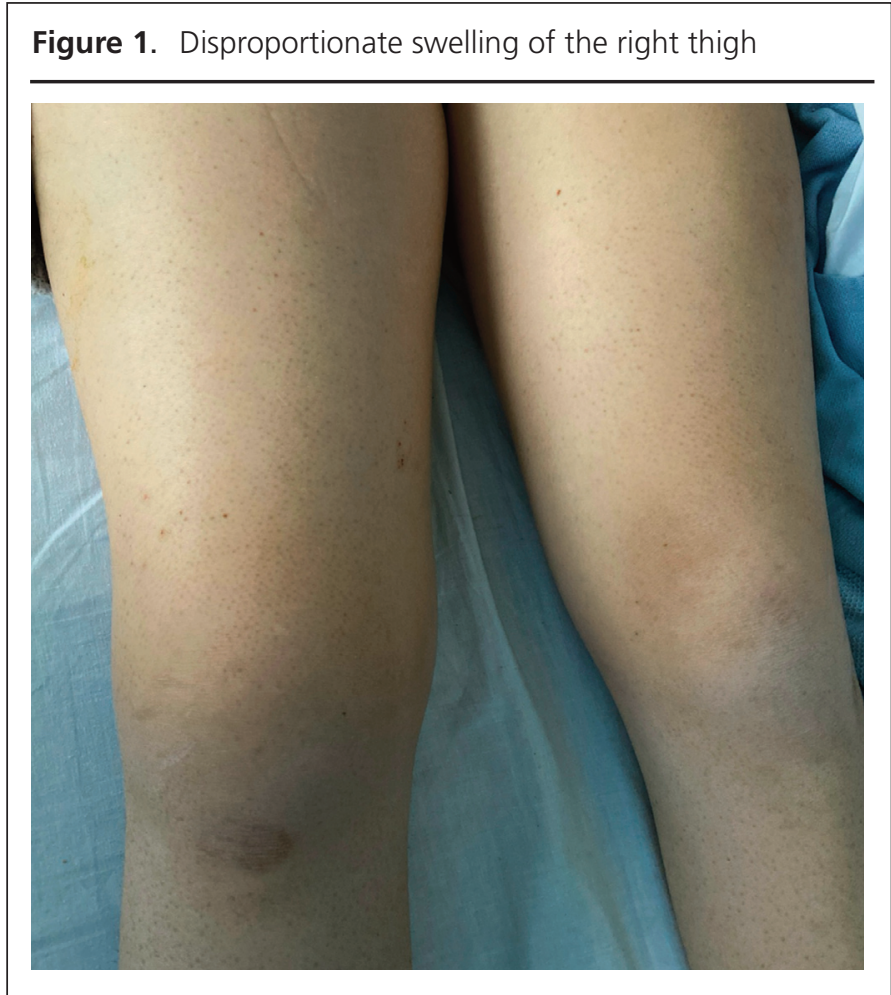

(Figure 1), very tender and warm to touch and movement limited due to pain. There was no erythema or inguinal lymphadenopathy and peripheral pulses were easily palpable.

\section{Investigations}

Admission blood tests showed a haemoglobin of $106 \mathrm{~g} / \mathrm{L}$ (normal range (NR) 115-160) and neutrophilia 9.64×109/L (NR 2-8) and Creactive protein (CRP) $133.7 \mathrm{mg} / \mathrm{L}$ (NR 0-5). Creatine kinase (CK) was normal at $172 \mathrm{U} / \mathrm{L}$ (NR 25-200) and D-dimer was marginally raised at $583 \mathrm{ng} / \mathrm{mL}$ (NR 0-500). Peripheral blood cultures and cultures from the peritoneal dialysis catheter were negative. Connective tissue antibody and myositis antibody screens were also negative.

Ultrasound Doppler of the right thigh excluded a focal collection and above-knee deep vein thrombosis (DVT) but did show focal muscle swelling (Figure 2). Magnetic resonance imaging (MRI) of both thighs confirmed unilateral extensive right medial compartment muscle swelling, myositis and ischaemia. This was most severe in portions of the sartorius, adductor longus and vastus intermedius. There was no focal soft tissue or osseous collection, marrow infarct or osteomyelitis (Figures 3 and 4). 
Figure 2. Transverse ultrasound image of the right thigh confirming focal area of muscle and subcutaneous swelling with heterogenous mixed echogenicity (arrow)

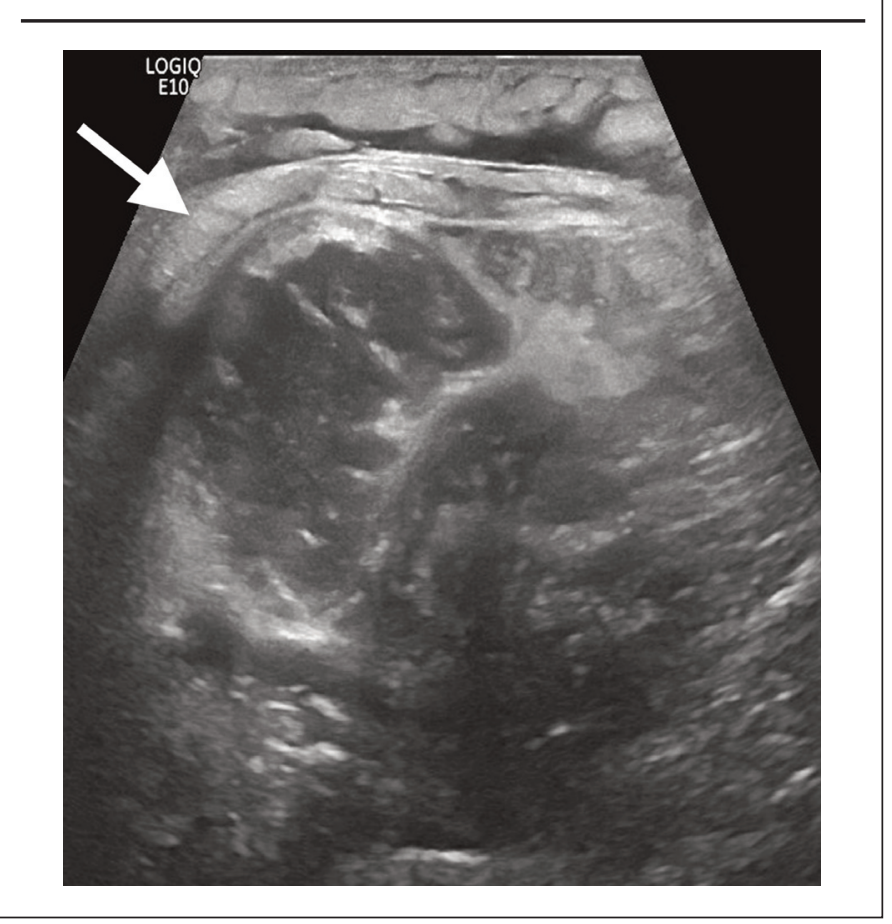

\section{Differential diagnosis and treatment}

At presentation the patient was given intravenous antibiotics for potential infective causes such as pyomyositis. Despite a prolonged course of broad-spectrum intravenous antibiotics, there was minimal symptomatic improvement. Microbiological investigations were consistently negative and there were no imaging findings to support an infective cause. The extent of pain prompted reviews from orthopaedic and vascular surgeons to exclude necrotising fasciitis, compartment syndrome and vascular insufficiency. DVT and focal collection were excluded on imaging. A rheumatology opinion was sought, and inflammatory or autoimmune myositis deemed unlikely. The long-standing history of poorly controlled diabetes with microvascular complications together with the clinical presentation and radiological findings pointed towards a diagnosis of DMN. Throughout her admission she had continual input from the diabetic specialist nurse and diabetologist recommending strict glycaemic control, analgesia and bedrest.

\section{Discussion}

DMN or diabetic muscle infarction is an uncommonly encountered complication of diabetes mellitus affecting patients with both types 1 and type 2. The mean age of onset ranges between 42 and 45 years and the time from diabetes diagnosis to the onset of DMN ranges from 15 to 20 years. ${ }^{2-4}$ DMN largely occurs in patients with poorly controlled diabetes with reported $\mathrm{HbA}_{1 \mathrm{c}}$ at diagnosis over $9 \%(75 \mathrm{mmol} / \mathrm{mol}),{ }^{4}$ usually in the presence of other microvascular complications. Diabetic nephropathy is the most common microvascular complication seen in DMN, reported
Figure 3. Axial T2-weighted fat-suppressed magnetic resonance image of the thighs, confirmed extensive near unilateral right upper thigh intramuscular swelling and oedema type signal, most severe within the medial compartment (arrow)

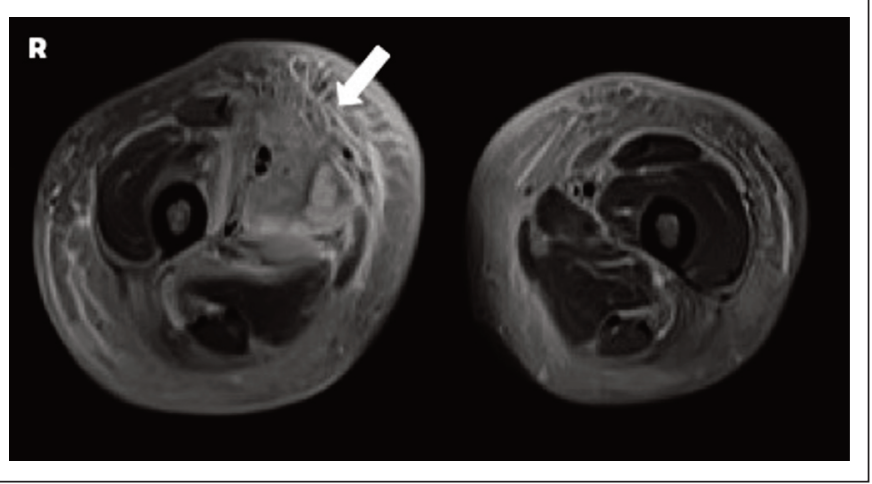

Figure 4. Coronal postcontrast-enhanced T1-weighted fat suppressed image confirming intense muscle, fascial and subcutaneous enhancement within the right upper medial thigh with focal central area of muscular non-enhancement (arrow)

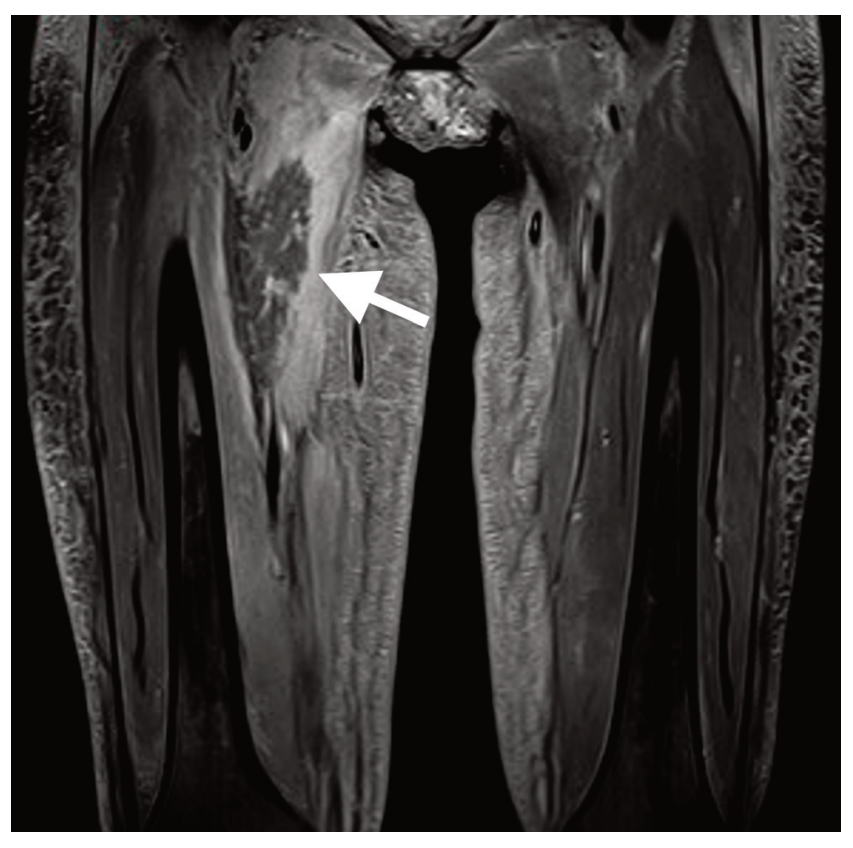

concurrently in 70-80\% of cases with a quarter of these dialysis dependent. ${ }^{2-5}$

Local pain and swelling are the usual presenting complaints and the quadriceps is the most commonly affected muscle group. Various pathogenic mechanisms have been postulated including atherosclerosis, diabetic microangiopathy, vasculitis with associated thrombosis, ischaemic-reperfusion injury and hypercoagulability state associated with diabetes mellitus. ${ }^{4,6}$ There are no diagnostic laboratory markers for DMN. White cell count, erythrocyte sedimentation rate, CRP and CK are all non-specific markers for DMN. ${ }^{5}$ 


\section{Key messages}

- Early involvement of the diabetes team at the time of admission is crucial in the management of patients with confirmed or suspected diabetic myonecrosis

- When managing patients presenting with suspected diabetic myonecrosis, detailed clinical history, review of diabetes control, physical examination, laboratory investigations and review of radiological images are imperative as the presentation can mimic appearances of necrotising fasciitis, compartment syndrome, vascular insufficiency, deep vein thrombosis, focal collection, inflammatory and autoimmune myositis

- A multidisciplinary approach to the diagnosis and management of diabetic myonecrosis is essential

MRI shows characteristic iso- to hypointensity on T1-weighted and high signal intensity on T2-weighted imaging of the affected muscle area with associated subcutaneous fat oedema. ${ }^{7,8}$ Muscle biopsy is not routinely used as a diagnostic tool to support the diagnosis of DMN due to observed increase in time to symptomatic improvement and procedure-associated complications. Histology is usually reserved for cases with atypical clinical presentations. ${ }^{4}$

Non-surgical management shows a statistically significant improvement in the time to recovery compared with surgical intervention such as excision of infarcted muscle (8.1 weeks and 13 weeks, respectively). ${ }^{9}$ Treatment with antiplatelet therapy or steroids has not been shown to be beneficial. ${ }^{9}$ To date there is no evidence from randomised controlled trials to support the optimal management for DMN.
A multidisciplinary approach to the management of DMN is indispensable. As described in our case, input from medical, surgical and radiological specialties was necessary to establish the diagnosis. Patient education and support is vital to improve understanding of the condition and to optimise glycaemic control as relapse of DMN is reported in up to $45 \%$ of cases. The mean mortality rate associated with DMN is $10 \%$ within 2 years of diagnosis with macrovascular events such as myocardial infarction, stroke or gangrene the predominant causes of death. ${ }^{3}$

Conflict of interest All authors have none to declare. Funding None.

\section{References}

1. Angervall L, Stener B. Tumoriform focal muscular degeneration in two diabetic patients. Diabetologia 1965;1:39-42. https://doi.org/ 10.1007/BF01338714

2. Trujillo-Santos AJ. Diabetic muscle infarction: an underdiagnosed complication of long-standing diabetes. Diabetes Care 2003;26(1):211-5. https://doi.org/10.2337/diacare.26.1.211

3. Kapur S, Brunet JA, McKendry RJ. Diabetic muscle infarction: case report and review. J Rheumatol 2004;31(1):190-4.

4. Horton WB, Taylor JS, Ragland TJ, et al. Diabetic muscle infarction: a systematic review. BMJ Open Diabetes Res Care 2015;3(1):1-8. https://doi.org/10.1136/bmjdrc-2015-000082

5. Yong TY, Khow KSF. Diabetic muscle infarction in end-stage renal disease: a scoping review on epidemiology, diagnosis and treatment. World J Nephrol 2018;7(2):58-64. https://doi.org/10.5527/wjn.v7.i2.58

6. Habib GS, Nashashibi M, Saliba W, et al. Diabetic muscular infarction: emphasis on pathogenesis. Clin Rheumatol 2003;22(6):450-1. https://doi.org/10.1007/s10067-003-0789-z

7. Huang BK, Monu JUV, Doumanian J. Diabetic myopathy: MRI patterns and current trends. Am J Roentgenol 2010;195(1):198-204. https://doi.org/10.2214/ajr.09.2494

8. Ganokroj P, Boonchaya-Anant P. Diabetic muscle infarction: rare complication with a distinct clinical manifestation. BMJ Case Reports 2019;12(4):1-4. https://doi.org/10.1136/bcr-2018-228480

9. Kapur S, McKendry RJ. Treatment and outcomes of diabetic muscle infarction. J Clin Rheumato/ 2005;11(1):8-12. https://doi.org/10.1097/ 01.rhu.0000152142.33358.f1 